\title{
POSITIONAL OBSERVATIONS OF COMPONENTS OF TRIPLE STARS FOR THE HIPPAR- COS AND HST MISSIONS
}

\author{
J.P. Anosova, V.V. Orlov \\ Leningrad University Astronomical Observatory \\ Bibliotechnaya Pl. 2 \\ 198904 Leningrad, USSR
}

ABSTRACT. This work has been made in accordance with the plan of the International Working Group "Double Stars: HIPPARCOS Input Catalogue Consortium." The precise coordinates for the components of the triple stars from the programs of the Astronomical Observatory (AO) of Leningrad State University (LSU) and the Uccle zone (declination from $+30^{\circ}$ to $+50^{\circ}$ ) are determined. The work has been made by two methods: (1) special astrometrical observations for about 70 triple stars; some colleagues from the observatories in Pulkovo, Nikolaev, and Goloseevo (USSR), as well as from Belgrade (Yugoslavia) have taken part in this work; (2) statistical treatment for about 200 triple stars, using the data of the catalogues ADS and WDS-all available positional observations of the relative coordinates of the components.

Also, the special observations of the triple stars in Pulkovo and Belgrade have been used, as well as the data from the catalogue by Ch. Worley from 1820 to 1988 . The old observations by W. Struve in 1820-1830 play an important role. The data obtained are used for the compilation of the observational programs of the AO of LSU by the HIPPARCOS and HST.

\section{PROBLEM OF USING LUNAR POSITIONAL OBSERVATIONS FOR DETERMINATION OF ZERO-POINTS OF FUNDAMENTAL STAR CATALOGUES}

\author{
V.A. Fomin \\ Central Astronomical Observatory, USSR Academy of Sciences \\ Pulkovo \\ 196140 Leningrad, USSR
}

ABSTRACT. The long series of meridian observations of the Moon can be used for the precise determination of the equinox- and equator-corrections of a star catalogue. Systematic errors of different charts of the lunar marginal zone used for the reduction of the lunar limb observations have no influence on the determination of the secular variations of the zero-points of the fundamental coordinate system.

From meridian observations of the lunar limb made during the interval 1923-1977 in Washington, Greenwich, Cape and Tokyo the following estimate is found for the correction to the right ascension system of the FK4 catalogue:

$$
\Delta \alpha(\mathrm{FK} 4)=-0.017 \pm 0.002-0.019 \pm 0.018(\mathrm{~T}-19.46) \mathrm{sec},
$$

which is in disagreement with the values used for the compilation of the FK5 catalogue. 